\title{
Performance Study of Block ACK and Reverse Direction in IEEE 802.11n using a Markov Chain Model
}

\author{
Md. Akbar Hossain, Nurul I Sarkar, Jairo Gutierrez, William Liu \\ School of Computer and Mathematical Sciences \\ Auckland University of Technology \\ Auckland 1010, New Zealand
}

\begin{abstract}
IEEE $802.11 \mathrm{n}$ networks are widely used in home and corporate network environments because they offer high-speed wireless Internet access at relatively low-cost. The 802.11n standard introduced several key features including Block acknowledgement (ACK) and reverse direction (RD) data transmission for enhanced system performance. An in-depth study of $802.11 \mathrm{n}$ system capacity for Block ACK mechanisms (both protected and unprotected) and RD data flows is required to assist optimum planning and design of such systems in view of the limited wireless channel capacity. In this paper we study the interdependencies of Block ACK and RD mechanisms using a discrete bi-directional Markov chain model under non-saturated traffic loads. We present a mathematical model to derive throughput, delay, and packet loss probability for both protected and unprotected Block ACKs under varying loads. We validate the model using MATLAB based numerical studies. Results obtained show that the combined effect of protected Block ACK and RD flows has a positive impact on system performance. However, unprotected Block ACK wastes transmission opportunity (TXOP) especially in collisions and therefore degrades the system performance. Our findings reported in this paper provide some insights into the performance of $802.11 \mathrm{n}$ with respect to Block ACK and RD methods. This study may help network researchers and engineers in their contribution to the development of next generation wireless LANs such as IEEE 802.11ac.
\end{abstract}


Keywords: Medium Access Control; Block ACK; Markov Chain; Reverse Direction; Distributed Coordination Function

\section{Introduction}

IEEE 802.11-based wireless local area networks (WLANs) are widely adopted in home and corporate networking environments due to their simplicity in operation, robustness, low cost, well-defined standards (e.g. 802.11a/b/g/n) and the user mobility offered by the technology. In the 802.11 standard, the distributed coordination function (DCF) is defined as a mandatory medium access control $(\mathrm{MAC})$ protocol and the point coordination function( $\mathrm{PCF})$ is optional [1]. The performance of DCF has been analyzed extensively using mathematical modeling and simulation 2, 3] 4]. In [2, Bianchi proposed a Markov chain model for a backoff mechanism to evaluate the throughput under saturated traffic and error free channel condition. Bianchi's work assume that packets will eventually transmitted regardless of the no. of retransmissions. However, a station (STA) will increase its contention window size after each failed transmission until it reaches the maximum backoff stage. Since the maximum backoff stage and retry limit are not equal, the contention window size remains the same and STA will continue retransmitting until it reaches a retry limit. If the subsequent transmission is not successful, the packet is discarded. The authors in [5], developed a Markov model which considers a finite retry limit for the transmission control protocol (TCP) over WLANs. They considered saturated traffic loads under ideal channel conditions. The extension of Bianchi's model was reported in [6] for finite load analysis. However, the maximum capacity of a wireless node is bounded by queue delays [7]. A finite load Markov model is presented in [7] by integrating a queue model as a new state with a Bianchi model assuming the STA queue is empty after successful transmission. All of these models are well studied for $802.11(\mathrm{a} / \mathrm{b} / \mathrm{g})$ networks. The fundamental goal of these models is to study the DCF protocol behavior under different channel and load conditions. The common thread of these studies is that the system performance can be 
enhanced by reducing MAC overheads.

The 802.11e standard [1] published in 2005, proposed a new MAC method called hybrid access method(HCF). A new ACK scheme is being introduced in the 802.11e standard known as BA. Unlike the traditional ACK scheme, an ACK is transmitted to reply to multiple data frames rather than per frame as in BA. Hence, the Markov model that has been developed for the traditional DCF protocol does not fit well with the BA scheme used to investigate the system throughput performance. Authors in [8] developed a Markov model for the BA and showed that a block with multiple frames can offers higher throughput than the traditional ACK based two-way or four-way transmission under saturated load and infinite retransmission conditions. But, when the frame consists of only one data frame it suffers from severe throughput degradation due to a couple of additional frames (e.g.BA request and BA). Moreover, it is assumed that data frames received with errors are considered a successful transmission, thus the contention window is reset. Unfortunately, according to the standard, receivers will not acknowledge the error data frame. Consequently, the sender has to retransmit the frame and increase the contention window if it does not reach a maximum contention value. Further enhancement of $[8]$ is reported in $[4$ by introducing a protected BA mechanism. The work reported in 4 inspired by further extensions of finite load conditions and integrating the RD features of 802.11n. Beside the BA scheme, frame aggregation mechanism is widely studied in recent literature to enhance the performance of 802.11n networks. In [9] discrete time Markov chain model is used to analyse the post backoff behavior due to frame aggregation under an error free environment. The performance study shows that, MAC service data unit (MSDU) outperforms the MAC protocol data unit (MPDU) as frame aggregation size becomes larger. An empirical study performs in [10] also confirms that a significant performance enhancement can be achieved when the frame aggregation and BA schemes are utilized. However, under an error prone channel frame aggregation mechanism experience severe throughput degradation and higher access delay due subframes size [11]. So far, we only consider the unidirectional data transmission. A bi-dimensional 
Markovian model presented in [12] shows that, bidirectional data transmission significantly enhance the overall network performance. Most of the previous studies on performance enhancement of 802.11n have focused on frame aggregation mechanisms. Very limited studies have actually analyzed the throughput performance of 802.11n under non-ideal channel conditions using Markov chains.

The main contribution of this paper is three fold. First, we present a simple Markov model to study the performance of $802.11 \mathrm{n}$ standard under nonsaturated traffic load. A detailed Markov chain model is developed by considering all possible constrains including load conditions, retry limits and channel state information. Second, we derived both Throughput and Packet delay for both the protected BA and non-protected BA schemes. Third, the effect of load conditions is analyzed in terms of packet loss probability. Moreover, an extensive MATLAB based numerical studies is presented to validate analytical model.

The rest of the paper is organized as follows: We describe the BA and RD mechanisms in Section 2. Section 3 presents a detailed discrete Markov model for $802.11 \mathrm{n}$ with a protected BA mechanism followed by three different subsections throughput, packet delay probability and mean delay (including MAC delay and Queue delay in consecutive subsections) analysis. A detailed numerical study including a comparative study of various mechanisms is presented in Section 4. A brief discussion in Section 5 ends the paper.

\section{Preliminaries}

\subsection{Block ACK Mechanism}

The Block ACK mechanism was first introduced in [1] to reduce the MAC overhead of 802.11e and later in 802.11n. The basic idea of the BA mechanism is to aggregate several ACK frames into a single frame. There are two different types of Block ACK mechanisms: Immediate (Im) and Delayed (D) Block ACK. A further extension of 802.11n for High Throughput(HT) operations classifies each of these Block ACK schemes in two subclasses: Protected 
and non-protected Block ACKs. The scope of this paper is limited to the ImBlock ACK scheme for both protected and non-Protected modes. In the ImBlock ACK scheme, transmitters and receivers are known as originators and recipients, respectively. To initialize the new acknowledgement policy, the originator and the recipient will exchange Add Block Acknowledgement(ADDBA) Request/Response frames. Afterwards, a data block with multiple data frames is transmitted from the originator to the recipient with Block ACK Request(BAR) at the end. The number of data frames in one data block is bounded by the receiver buffer size. The recipient sends a Block Acknowledgement (BA) frame for the the entire data block. Figure1(a) shows the protected Block ACK channel access mechanism. In protected Block ACK, before transmitting an entire data burst, the originator will transmit a single data frame and wait for an ACK from the recipient. After the successful reception of an ACK frame, the originator initiates the transmission opportunity (TXOP) period to transmit the data burst. Therefore, if there is an error or channel collision,this problem would only be experienced by the first data frame or ACK frame. This concept is most likely an RTS/CTS based four-way handshake (shown in Fig. 1(c)) mechanism except when using a special RTS/CTS frame where as the non-protected Block ACK mechanism is based on a two-way handshake mechanism. The time diagram of the non-protected Block ACK mechanism is depicted in Fig.1. In terms of throughput as a performance metric, protected Block ACK should outperform the non-protected Block ACK scheme by reducing the wasted time due to collision or channel errors.

\subsection{Reverse Direction}

We propose an efficient reverse direction (RD) data exchange protocol to improve QoS support and overall efficiency of the IEEE 802.11n standard for high rate physical layer. The RD protocol provides mechanisms that significantly reduce the MAC-overhead while retaining full compatibility with legacy systems. Figure 1(c) illustrates the RD scheme with a block ACK mechanism. In this specified transmission, the receiver may request a reverse data transmission in 


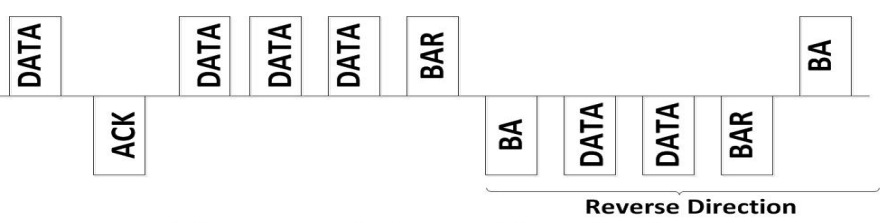

(a) Protected Block ACK Mechanism

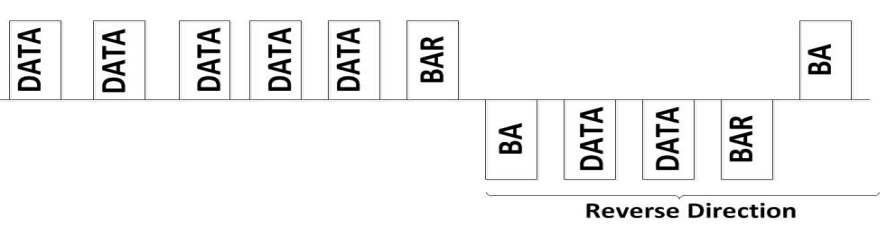

(b) Non-Protected Block ACK Mechanism

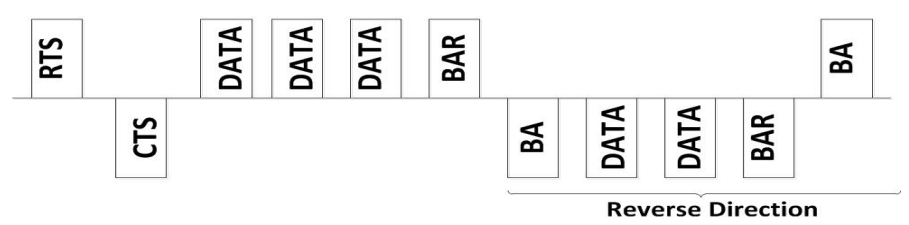

(c) RTS/CTS Based Block ACK Mechanism

Figure 1: Various Block ACK mechanisms with Reverse Direction

the CTS control frame. This allows the transportation of data frames and also aggregates frames, in both directions, in one Transmission Opportunity (TXOP) period. Until now, when the sender STA is allocated with a TXOP, it informs surrounding STAs about how long the wireless medium will be engaged. Hence, $\mathrm{RD}$ achieves better results by supporting "on-demand" bi-directional data flows using the existing handshake protocol without any additional control frames. Furthermore, it reduces block transmission overhead by eliminating the short interframe space(SIFS) for transmission in both directions and relies on a single block acknowledgement frame. Previously, for each uni-directional data transfer, the initiating station needed to contend for the channel in a contention-based wireless medium. With $\mathrm{RD}$, the other stations are essentially allowed to send information back once the transmitting station has attained a TXOP. Therefore, two roles need to be identified: $\mathrm{RD}$ initiator and $\mathrm{RD}$ responder. The RD initiator sends its permission to the $\mathrm{RD}$ responder due to a Reverse Direction 
Grant (RDG) in the RDG/More physical layer convergence protocol (PLCP) protocol data unit (PPDU) field of the high throughput (HT) control field. The RD mechanism facilitates the data transmission from both sides (sender and receiver) without further contending for the medium and reducing the number of contentions by a factor of 1.5 to 2 [13]. Moreover, the proposed RD mechanism reduces the overall MAC overhead associated with collision detection and medium protection. A similar concept for a RD mechanism was presented in [14, where receiver data is piggybacked with a Block ACK to the sender. Obviously this modification increases the throughput for the system but, as far as we can ascertain, an overall performance analysis including error and MAC delay analysis are still not found in the recent literature.

\section{Markov Model for IEEE 802.11n with Protected BLOCK ACK}

In this section, an analytical model is proposed to evaluate the performance of 802.11n under non-saturated load conditions. A two dimensional Markov model is developed to derive the channel throughput and end-to-end delay of successful data transmissions. To simplify the mathematical model we made the following assumptions.

(i) Finite number of stations

(ii) Unsaturated load i.e. there is a certain probability that the transmission queue is empty

(iii) Channel is prone to errors

(iv) No hidden terminals

(v) Packets are destroyed only through collisions exceeding the retry limit

(vi) Packets are of equal length

The proposed model also takes into consideration both basic handshake (twoway) and RTS/CTS handshake (four-way)-based channel access schemes. Developing a Markov model to evaluate the DCF performance of WLANs was first shown by using the Bianchi Model 2] under saturated loads with ideal channel 
conditions. Unfortunately Markov Models developed for $802.11 \mathrm{a} / \mathrm{b} / \mathrm{g}$ are not substantial enough to explain the $802.11 \mathrm{n}$ behavior which has distinct features such as Protected Block ACK and Reverse Direction Data flows. In our model, we derive both the throughput and end-to-end delays by taking into account protected Block ACKs and Reverse Direction data flows in non-saturated load conditions.

The fundamental access method of 802.11 is called distributed coordination function (DCF) which is based on carrier sense multiple access with collision avoidance (CSMA/CA). For a STA to transmit, it senses the medium to determine if another STA is in transmission. According to DCF distributed protocol there must be a minimum time gap to identify contiguous frame sequences. Therefore, a transmitting STA must ensure that the medium is idle for this period before attempting to transmit. If the medium continues to be busy, the STA defers until the end of the current transmission. After deferral, or prior to attempting to transmit again immediately after a successful transmission, the STA selects a random backoff interval and decrements the backoff interval counter while the medium is idle. A transmission is successful if an acknowledgement (ACK) frame is received from the targeted STA (unicast) or when a multicast frame is sent completely. A refinement method may be used to further reduce collisions: the transmitting and receiving STAs exchange short control frames, request to send (RTS) and clear to send (CTS). Let $n$ be the number of stations (STAs)in a WLAN contending for channel access. Let $b(t)$ and $s(t)$ be the stochastic processes representing the backoff counter and backoff stages at time $t$ respectively. Hence according to DCF, the $b(t)$ value is decremented at the start of every idle slot and a contending station wins the channel when it reaches to zero. After successful transmissions if the STA has more data to send a new value would be set for $b(t)$. As the counter value of $k=b(t)$ is chosen to be uniformly distributed over $k \in\left[0, C W_{i}\right]$, where $C W_{i}$ stands for the contention window size, there is a chance that two STAs end up with the same $b(t)$ values and transmit data simultaneously. This is called a collision. In order to avoid further collisions, the collided STAs will generate new $b(t)$ values 
determined by:

$$
C W_{i}=\left\{\begin{array}{l}
2^{i} C W_{m i n} ; i \leq m^{\prime}, \\
2^{m^{\prime}} C W_{m i n}=C W_{m} ; i>m^{\prime}
\end{array}\right.
$$

Where $C W_{i}$ is an initial size for the contention window and $m^{\prime}$ is a maximum number by which the contention window can be doubled. In this model, $m$ is used to resemble the maximum backoff stage. According to the IEEE 802.11 standard, the value $m$ could be larger than $m^{\prime}$, while the $C W$ will hold after that. Therefore, every STA is modeled by a pair of integers $(i, k)$. At the very first time, the backoff stage $i=s(t)$ starts at 0 and is increased by 1 everytime transmissions collide. Once the $C W$ reaches $C W_{m}$, it will remain at this value until it is reset. That means STA will keep transmitting the packet till it reaches the retry limit. If the transmission is still unsuccessful the packet will be dropped. The two-dimensional process $(s(t), b(t))$ will be analyzed with an embedded Markov chain (in steady state) at time instants at which the channel state changes. Let $(\mathrm{i}, \mathrm{k})$ denote the state of this process. At each stage the STA is described by $i, k$ where $i$ stands for the backoff stage and $k$ stands for the backoff delay which takes any value in the range of $\left[0, C W_{i}-1\right]$. Let, $p_{f}$ denote the frame failure transition probability from one stage to another (e.g. from row $i-1$ to row $i$ in Fig, 2). It is also the probability of an unsuccessful (re)transmission attempt seen by a STA as its frame is being transmitted on the channel. The unsuccessful (re)transmission attempt can happen due to the collision of this station with at least one of the $n-1$ remaining stations, occurring with probability $p_{\text {coll }}$, where $p_{\text {coll }}$ is

$$
p_{\text {coll }}=1-(1-\tau)^{n-1}
$$

and/or by having a frame with errors, occurring with probability $p_{\text {err }}$ (due to channel fading and/or noise). Since both events are independent, the probability p can be expressed as:

$$
p_{f}=1-\left(1-p_{\text {coll }}\right)\left(1-p_{\text {err }}\right)=p_{\text {coll }}+p_{\text {err }}-p_{\text {coll }} p_{\text {err }}
$$




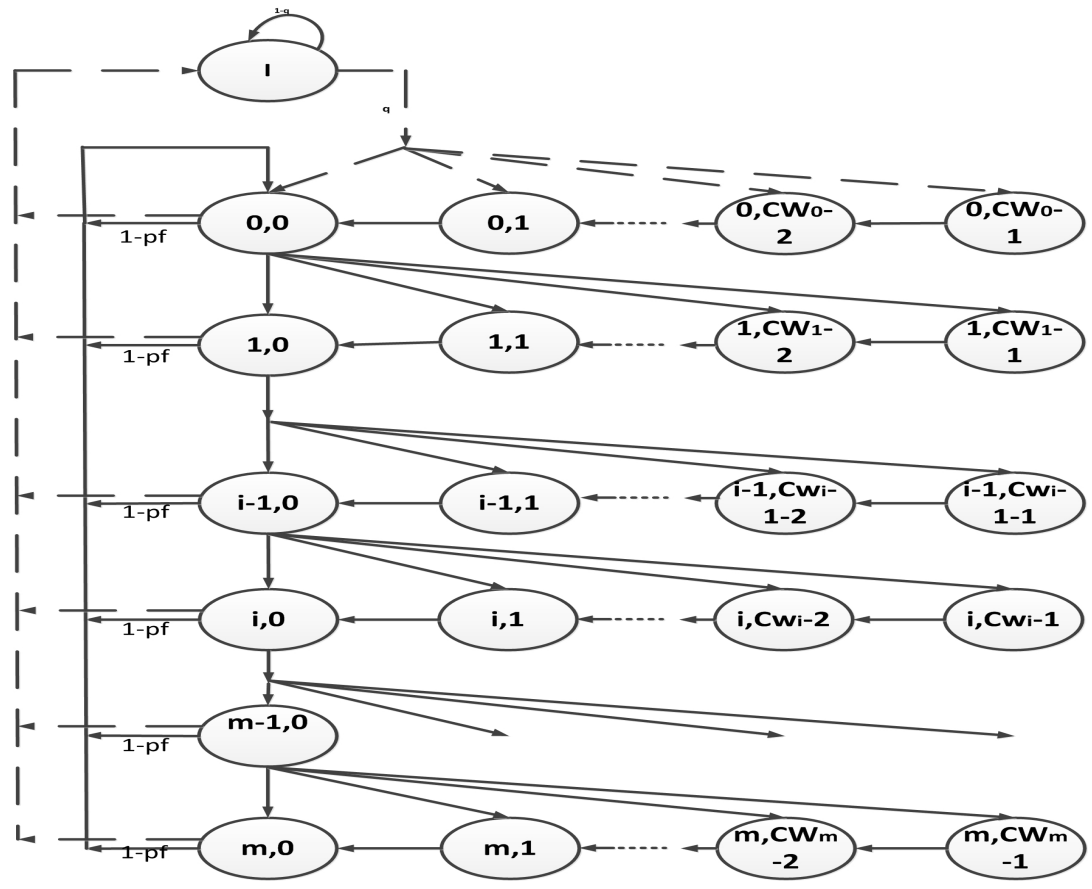

Figure 2: Two Dimensional Markov Chain Model for 802.11n backoff

In case of an unsuccessful transmission attempt, after the backoff timer expires in state $(i, 0)$, the station moves to any state on row $i(i, k)$ with probability $p_{f} / C W_{i}$. Following a successful transmission (occurring with conditional probability $p_{f}$ ) while the observed station is in stage $i \in(0, m)$, a new packet is admitted in the queue, the station returns to backoff stage 0 , and its backoff timer uniformly selects any integer value in the range $\left(0, C W_{0}-1\right)$ with probability $\left(1-p_{f}\right) / W_{0}$. If the station reaches backoff stage $\mathrm{m}$, and once its backoff timer reaches 0 , its frame can be successfully or unsuccessfully transmitted. In both cases, a new frame is admitted in the queue and the station returns to backoff stage 0 ,and its backoff timer is uniformly chosen in the range $\left(0, C W_{0}-1\right)$ with probability $1 / C W_{0}$. After a successful transmission or after $m^{\text {th }}$ failed retransmissions, it is assumed that the buffer of the transmitting station is not empty with probability $q$ and empty with probability $1-q$. 
Based on the above discussion the transition probabilities of the Markov process are determined as follows:

1. $P(i, k \mid i, k+1)=1 ; \quad k \in\left[0, C W_{i}-2\right], i \in[0, m]$

The station's backoff timer is decremented from $k+1$ to $k$ at fixed $i$ backoff stages, i.e. the station has detected an idle slot, so the channel is idle.

2. $P(0, k \mid i, 0)=q\left(1-p_{f}\right) / C W_{0} ; \quad k \in\left[0, C W_{0}-1\right], i \in[0, m]$ After a successful transmission with probability $1-p_{f}$, it is assumed that there is a new packet in the transmitting STA buffer with probability $q$ to start a new transmission at backoff stage 0 .

3. $P(i, k \mid i-1)=p_{f} / C W_{i} ; \quad k \in\left[0, C W_{i}-1\right], i \in[1, m]$

The station's backoff timer is changed from 0 to $k$ and the backoff stage is changed from $i-1$ to $i$. The probability of this event equals: $\operatorname{Pr}$ (transmission is unsuccessful and number $k$ was randomly chosen to initiate the backoff timer at stage $i)=\operatorname{Pr}($ transmission is unsuccessful $) \cdot \operatorname{Pr}($ number $k$ was randomly chosen to initiate the backoff timer at stage $i$ ). The probability of unsuccessful transmission equals $p_{f}$ and the probability that number $k$ was randomly chosen to initiate the backoff timer at stage $i$ equals $1 / C W_{i}$.

4. $P\left(m, k \mid m^{\prime}, 0\right)=p_{f} / C W_{m} ; \quad k \in\left[0, C W_{m}-1\right]$

The probability of unsuccessful transmission equals $p_{f}$. The probability that number $k$ was randomly chosen to initiate the backoff timer at stage $m$ (maximum backoff stage) equals $1 / C W_{m}$ till reaching the retry limit.

5. $P(I \mid i, 0)=(1-q)\left(1-p_{f}\right) ; \quad i \in[0, m]$

This equation represents the practical scenario i.e. unsaturated traffic condition where the transmitting STA's buffer is empty with probability $1-q$ after a successful transmission. Hence the station will not contend for a channel till the new packet has arrived.

6. $P(I \mid I)=1-q$

This stage is known as the Idle stage, when STA doesn't have any packet in its buffer to transmit.

7. $P(0, k \mid I)=q / C W_{0} ; \quad k \in\left[0, C W_{0}-1\right]$

This means a new packet arrives at the buffer with probability $q$ and 
starts a new transmission by using a randomly chosen delay $k$ to initiate the backoff counter at stage 0 with probability $1 / C W_{0}$

8. $P(0, k \mid m, 0)=q / C W_{0} ; \quad k \in\left[0, C W_{0}-1\right]$

The transition probability mentioned in the previous equation could happen when a packet is discarded due to a failure to transmit within the retry limit. Here it is assumed that there is at least a packet in the buffer.

9. $P(I \mid m, 0)=1-q$

If the buffer is empty with probability $1-q$ after the retry limit.

Let the stationary distribution of the chain be $b_{i, k}=\lim _{t \rightarrow \infty} P\{s(t)=$ $i, b(t)=k\}, i \in(0, m), k \in\left(0, C W_{i}-1\right)$, denoting the probability of the station to be in $\operatorname{state}(i, k)$. The probability of the station to be in state $(i, 0)$ can be expressed as a $n$ stage transition probability as follows:

$$
\begin{gathered}
b_{i-1,0} * p_{f}=b_{i, 0} \quad 0<i \leq m \\
b_{i, 0}=p_{f}^{i} b_{0,0} \quad 0 \leq i \leq m
\end{gathered}
$$

Since the chain is regular, for each $k \in\left[0, C W_{i}-1\right]$

$$
b_{i, k}=\frac{C W_{i}-k}{C W_{i}}\left\{\begin{array}{l}
q\left(1-p_{f}\right) \cdot \sum_{i=0}^{m-1} b_{i, 0}+b_{m, 0}+q \cdot b_{I} \\
p_{f} b_{i-1,0} \quad 0<0 \\
0<i \leq m
\end{array}\right.
$$

Now

$$
\begin{gathered}
b_{I}=(1-q)\left(1-p_{f}\right) \sum_{i=0}^{m} b_{i, 0}+(1-q) \cdot b_{I} b_{I}-(1-q) b_{I}=(1-q)\left(1-p_{f}\right) \sum_{i=0}^{m} b_{i, 0} \\
b_{I}=\frac{(1-q)\left(1-p_{f}\right)}{q} \sum_{i=0}^{m} b_{i, 0}
\end{gathered}
$$


Now from equations 6 and 7

$$
\begin{aligned}
& q\left(1-p_{f}\right) \cdot \sum_{i=0}^{m-1} b_{i, 0}+q \cdot b_{I}+b_{m, 0} \\
& =q\left(1-p_{f}\right) \cdot \sum_{i=0}^{m-1} b_{i, 0}+q \cdot b_{I}+b_{m, 0} \\
& =\left(1-p_{f}\right)\left[q \sum_{i=0}^{m-1} b_{i, 0}+(1-q) \cdot \sum_{i=0}^{m} b_{i, 0}\right]+b_{m, 0}
\end{aligned}
$$

Utilizing the relation established in Eq. 44 and make use of $\sum_{i=0}^{m} b_{i, 0}=\frac{b_{0,0}}{1-p_{f}}$, Eq. (6) can be written as

$$
b_{i, k}=\frac{C W_{i}-k}{C W_{i}} b_{i, 0} i \in(0, m), k \in\left(0, C W_{i}-1\right)
$$

Now by using the normalization condition for stationary distribution equation:

$$
\begin{aligned}
1 & =\sum_{i=0}^{m} \sum_{i=0}^{m-1} b_{i, 0} b_{i, k}+b_{I} \\
& =\sum_{i=0}^{m} b_{i, 0} \sum_{i=0}^{m-1} b_{i, 0} b_{i, k} \frac{C W_{i}-k}{C W_{i}}+b_{I} \\
& =\sum_{i=0}^{m} p_{f}^{i} b_{0,0} \frac{C W_{i}-k}{2}+b_{I} \\
& =\frac{b_{0,0}}{2} \sum_{i=0}^{m} p_{f}^{i}\left(2^{i} C W+1\right)+b_{I} \\
& =\frac{b_{0,0}}{2}\left(\sum_{i=0}^{m}\left(2 p_{f}\right)^{i}+\sum_{i=0}^{m} p_{f}^{i}\right)+b_{I}
\end{aligned}
$$

Now for $m \leq m^{\prime}$ (i.e the retry limit is within the range of the maximum backoff stage)

$$
\begin{aligned}
& =\frac{b_{0,0}}{2}\left(\frac{\left(C W\left(1-\left(2 p_{f}\right)^{m+1}\right)\right)}{1-2 p_{f}}+\frac{1-p_{f}^{m+1}}{1-p_{f}}\right)+\frac{(1-q)\left(1-p_{f}\right)}{q} \sum_{i=0}^{m} b_{i, 0} \\
& =\frac{b_{0,0}}{2}\left(\frac{\left(C W\left(1-\left(2 p_{f}\right)^{m+1}\right)\right)}{1-2 p_{f}}+\frac{1-p_{f}^{m+1}}{1-p_{f}}\right)+\frac{(1-q)\left(1-p_{f}^{m+1}\right)}{q} b_{0,0} \\
1 & =\frac{b_{0,0}}{2}\left(\frac{\left(C W\left(1-\left(2 p_{f}\right)^{m+1}\right)\right)}{1-2 p_{f}}+\frac{1-p_{f}^{m+1}}{1-p_{f}}\right)+\frac{2(1-q)\left(1-p_{f}^{m+1}\right)}{q} \\
& =\frac{b_{0,0}}{2}\left(\frac{A}{q\left(1-2 p_{f}\right)\left(1-p_{f}\right)}\right)
\end{aligned}
$$


where $A=q\left(1-p_{f}\right) C W\left(1-\left(2 p_{f}\right)^{m+1}\right)+q\left(1-2 p_{f}\right)\left(1-p_{f}^{m+1}\right)+2\left(1-2 p_{f}\right)(1-$ $\left.p_{f}\right)(1-q)\left(1-p_{f}^{m+1}\right)$

$$
b_{0,0}=\frac{2 q\left(1-2 p_{f}\right)\left(1-p_{f}\right)}{A}
$$

Now the probability of transmission at any random slot can be written as

$$
\begin{aligned}
\tau & =\sum_{i=0}^{m} b_{i, 0} \\
& =\frac{1-p_{f}^{m+1}}{1-p_{f}} \cdot b_{0,0} \\
\tau & =\frac{2 q\left(1-2 p_{f}\right)\left(1-p_{f}^{m+1}\right)}{A} ; \quad m \leq m^{\prime}
\end{aligned}
$$

For $m \geq m^{\prime}$ (i.e retry limit is greater than maximum backoff stage)

$$
\begin{aligned}
& =\frac{b_{0,0}}{2}\left(\sum_{i=0}^{m^{\prime}} C W_{i}+\sum_{i=m^{\prime}+1}^{m} p_{f}^{i} C W^{i}+\sum_{i=0}^{m} p_{f}^{i}\right)+b_{I} \\
& =\frac{b_{0,0}}{2}\left(\frac{C W\left(1-2 p_{f}^{m^{\prime}+1}\right)\left(1-p_{f}\right)+\left(1-2 p_{f}\right)\left(1-p_{f}^{m+1}\right)+2^{m^{\prime}} C W p_{f}^{m^{\prime}+1}\left(1-2 p_{f}\right)\left(1-p_{f}^{m-m^{\prime}}\right)}{\left(1-2 p_{f}\right)\left(1-p_{f}\right)}\right)+ \\
& +\frac{(1-q)\left(1-p_{f}^{m+1}\right)}{q} b_{0,0} \\
b_{0,0} & =\frac{2 q\left(1-p_{f}\right)\left(1-2 p_{f}\right)}{B} \\
B= & q\left[C W\left(1-\left(2 p_{f}\right)^{m^{p r i m e}}+1\right)\left(1-p_{f}\right)+\left(1-2 p_{f}\right)\left(1-p_{f}^{m+1}\right)+\right. \\
+ & \left.2^{m^{\prime}} C W p_{f}^{m^{\prime}+1}\left(1-2 p_{f}\right)\left(1-p_{f}^{m-m^{\prime}}\right)\right]+2(1-q)\left(1-p_{f}^{m+1}\right)\left(1-2 p_{f}\right)\left(1-p_{f}\right)
\end{aligned}
$$

Thus

$$
\begin{aligned}
\tau & =\sum_{i=0}^{m} b_{i, 0} \\
& =\frac{1-p_{f}^{m+1}}{1-p_{f}} \cdot b_{0,0} \\
\tau & =\frac{2 q\left(1-2 p_{f}\right)\left(1-p_{f}^{m+1}\right)}{B} ; \quad m \geq m^{\prime}
\end{aligned}
$$

So far, $p_{f}$ is still unknown but can be solved by using Eq.(3), where $p_{\text {coll }}=$ $1-(1-\tau)^{n-1} \cdot p_{\text {err }}$ in Eq. (3), stands for the frame error probability(FER)of a 
MAC data frame or an ACK frame for the given STA which can be expressed as an independent events as follows:

$$
\begin{aligned}
p_{e r r} & =1-\left(1-F E R_{\text {data }}\right)\left(1-F E R_{a c k}\right) \\
& =F E R_{\text {data }}+F E R_{a c k}-F E R_{\text {data }} \cdot F E R_{a c k}
\end{aligned}
$$

We can rewrite FER with respect to STA's mobility, receiver fading margin, transmission carrier frequency, and frame duration [15].

$$
F E R=1-\exp \left(-\rho-f_{d} \sqrt{2 \pi \rho T_{p}}\right)
$$

Where $\rho$ is the fading margin decided by the receiver structure, $f_{d}$ is the maximum doppler frequency calculated from the STA speed and the carrier frequency,and $T_{p}$ represents frame duration. Hence, Eq. 3 becomes:

$$
p_{f}=1-(1-\tau)^{n-1}\left(1-F E R_{\text {data }}\right)\left(1-F E R_{a c k}\right)
$$

In this analysis, the unsaturated traffic behavior is characterized by defining a $\operatorname{MAC}$ queue $(q)$ in equations 11 and $13, q$ can be defined as the probability that there is at least one packet to be transmitted in the STA queue. Assume, the packet arrival rate at each STA buffer from upper layer is $\lambda \mathrm{pkt} / \mathrm{sec}$ and $\mu$ represent the packet processing rate assuming that the queue has a length of $K$. By using a $M / M / 1 / K$ queueing model[15], the probability $q$, that there is at least one packet in the queue is:

$$
q=1-\frac{1-\frac{\lambda}{\mu}}{1-\left(\frac{\lambda}{\mu}\right)^{K+1}}
$$

Here $\mu=\frac{\tau_{s}\left(1-\tau_{s}\right)^{n-1}}{\overline{\sigma_{s}}} . \tau_{s}$ and $\overline{\sigma_{s}}$ are packet transmission probabilities at any random slot and average slot times at saturated load conditions. Equations 15[11, and 13 represent a non-linear system with two unknown parameters $\tau$ and $p_{f}$ having single solution which can be solved numerically.

\subsection{Throughput Analysis}

Let $P_{t r}$ be the probability that at least one station transmits a packet in a randomly selected slot time with probability $\tau$ and $P_{s}$ is the conditional probability that an occurring packet transmission is successful. For a WLAN with $n$ 
contending stations, the probabilities $P_{t r}$ and $P_{s}$ can be written as

$$
\begin{aligned}
& P_{t r}=1-(1-\tau)^{n} \\
& P_{s}=\frac{n \tau(1-\tau)^{n-1}}{1-(1-\tau)^{n}}
\end{aligned}
$$

Considering that a random slot is empty with probability $\left(1-P_{t r}\right)$ and that it contains a successful transmission with probability $P_{t r} P_{s}$, a collision with probability $P_{t r}\left(1-P_{s}\right)$ and $P_{e r r}$ in 14 is the probability of a channel access failure due to channel error. According to [3], the throughput $S$ is defined as a ratio of successfully transmitted payload size over a randomly chosen slot duration:

$$
S=\frac{P_{t r} \cdot P_{s} \cdot\left(1-P_{e r r}\right) E\left[P_{T}\right]}{\left(1-p_{t r}\right) \sigma+P_{t r} P_{s}\left(1-P_{e r r}\right) T_{s}+P_{t r}\left(1-P_{s}\right) T_{c}+P_{t r} P_{s} P_{e r r} T_{e}}
$$

where $\sigma$ is the backoff slot duration, $T_{s}$ is the average time that the channel is captured with successful transmission, $T_{c}$ is the average time that the channel is captured by stations which collide and $T_{e}$ is the average wasted time due to a channel access failure caused by channel error due to mobility and fading effects. Hence the duration of a channel slot is the period of time the channel stays in one state:idle, fail including collision and error and finally success. Let $T_{\text {data }}, T_{a c k}, T_{r t s}, T_{c t s}, T_{b a r}, T_{b a}, T_{h o b}$ and $T_{h a c k}$ be the transmission times (measured in microseconds) of an MPDU, an ACK frame, a RTS frame, a CTS frame, a BlockAckReq (BAR) frame, a BlockAck (BA), a Head of Brust(HOB) and a Head of $\mathrm{ACK}(\mathrm{HACK})$ frame, respectively. As in [4] it is also assumed that the frame could be corrupted due to either collision or channel error that leads to retransmission and increment of the backoff stage. When using the protected block ack mechanism, a data burst transmission cannot be initiated at TXOP if there is an error or collision in the HOB or HACK frames [1]. Hence, a data 
burst duration $T_{s}, T_{e}, T_{c}$ is given by $T_{s}=\left\{\begin{array}{l}T_{a c k}+T_{s i f s}+\left(T_{d a t a}+T_{s i f s}\right) \cdot B+T_{b a r}+T_{s i f s}+T_{b a}+T_{R D} ; \\ \text { Protected Block ACK Scheme } \\ \left.T_{\text {data }}+T_{T_{s i f s}}\right) \cdot B+T_{b a r}+T_{s i f s}+T_{b a}+T_{R D}+T_{\text {difs }} ; \\ \text { Unprotected Block ACK Scheme } \\ T_{r t s}+T_{c t s}+2 T_{\text {sifs }}+\left(T_{\text {data }}+T_{\text {sifs }}\right) \cdot B+T_{b a r}+T_{s i f s}+T_{b a}+T_{R D}+T_{\text {difs }} ; \\ \text { RTS/CTS scheme }\end{array}\right.$

$T_{e}=\left\{\begin{array}{l}T_{\text {hob }}+T_{\text {eifs }}+\left(T_{\text {sifs }}+T_{\text {hack }}\right) \times \frac{1-F E R_{h o b}}{F E R_{\text {hob }}+F E R_{\text {hack }}-F E R_{\text {hob }} F E R} \\ \text { Protected Block ACK Scheme } \\ \left(T_{\text {data }}+T_{T_{s i f s}}\right) \cdot B+T_{\text {bar }}+T_{\text {sifs }}+T_{b a}+T_{R D}++T_{\text {difs }} \\ \text { Unprotected Block ACK Scheme } \\ T_{r t s}+T_{\text {eifs }}+\left(T_{\text {sifs }}+T_{c t s}\right) \times \frac{1-F E R_{r t s}}{F E R_{r t s}+F E R_{c t s}-F E R_{r t s} F E R_{c t s}} \\ \text { RTS/CTS scheme }\end{array}\right.$

$$
T_{c}=\left\{\begin{array}{l}
T_{h o b}+T_{\text {eifs }} \\
\text { Protected Block ACK Scheme } \\
\left(T_{\text {data }}+T_{T_{\text {sifs }}}\right) \cdot B+T_{b a r}+T_{\text {sifs }}+T_{b a}+T_{R D}++T_{\text {difs }} \\
\text { Unprotected Block ACK Scheme } \\
T_{r t s}+T_{\text {eifs }} \\
\text { RTS/CTS scheme }
\end{array}\right.
$$

$$
T_{R D}=T_{\text {sifs }}+\left(T_{\text {data }}+T_{\text {sifs }}\right) \cdot B_{R D}+T_{\text {bar }}+T_{\text {sifs }}+T_{b a}(\text { ReverseDirection })
$$

All of the above mentioned time equations consider RD transmissions which can easily be unplugged to calculate the throughput without RD. According to [4], 
it is also assumed that the HOB frame is always successful to gain the TXOP. Thus $E\left[P_{T}\right]$ is given by

$$
E\left[P_{T}\right]=\left\{\begin{array}{l}
L \cdot(E[B]-1)\left(1-F E R_{\text {data }}\right)+L \cdot\left(E\left[B_{R D}\right]\right)\left(1-F E R_{\text {data }}\right)+L \\
\text { Protected Block ACK with RD } \\
L \cdot(E[B]-1)\left(1-F E R_{\text {data }}\right)+L ; \\
\text { Protected Block ACK without RD } \\
L \cdot(E[B])\left(1-F E R_{\text {data }}\right) ; \\
\text { RTS/CTS Method and Unprotected Block ACK } .
\end{array}\right.
$$

\subsection{Packet Drop Probability}

The packet drop probability is the probability that a packet is dropped when the retry limit is reached. Moreover, a packet may be dropped when the sending queue is full. Hence the total packet drop probability is the sum of both of these events.

\subsubsection{Packet Drop Due to Retry Limit}

A packet is found in the last backoff stage $m$ if it encounters $m$ collisions in the previous stages and it is eventually discarded or dropped. Thus, the packet drop probability due to reaching the retry limit can be written as a function of the last backoff stage:

$$
\begin{aligned}
P_{\text {drop }} & =\frac{b_{m, 0}}{b_{0,0}} p_{f} \\
& =p_{f}^{m} \cdot p_{f} \\
& =p_{f}^{m+1} \\
& =\left[1-\left(1-p_{f}\right)(1-\tau)^{n-1}\right]^{m+1}
\end{aligned}
$$

\subsubsection{Packet Drop Due to Queue}

Let us consider the $M / M / 1 / K$ queue system, where there are $K$ frames in the system shown in Fig. 3 . Now, by using one of the balanced equations, the steady state probability can be written as 


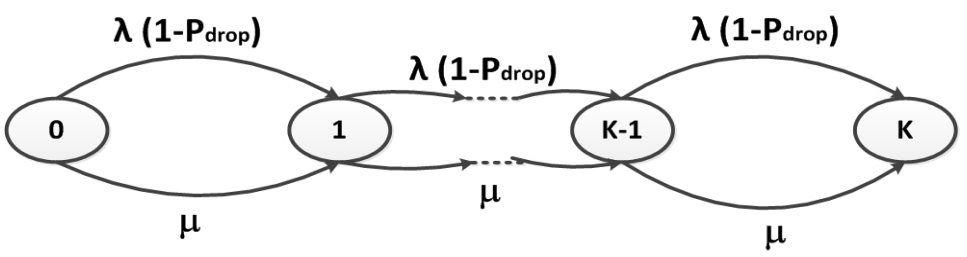

Figure 3: M/M/1/K Queue Model

$$
\begin{aligned}
\lambda\left(1-p_{\text {drop }}\right) p_{0} & =\mu p_{1} \\
p_{1} & =\frac{\lambda\left(1-p_{\text {drop }}\right)}{\mu} p_{0} \\
p_{1} & =\rho p_{0} ; \text { where }, \rho=\frac{\lambda\left(1-p_{\text {drop }}\right)}{\mu}
\end{aligned}
$$

Similarly it can be shown that

$$
\begin{aligned}
p_{n} & =\rho^{n} p_{0} ; n=0,1,2, \ldots . . K \\
\sum_{n=0}^{K} p_{n} & =1 \Rightarrow p_{0}=\frac{1-\frac{\lambda\left(1-p_{\text {drop }}\right)}{\mu}}{1-\left(\frac{\lambda\left(1-p_{\text {drop }}\right)}{\mu}\right)^{K+1}}
\end{aligned}
$$

Note that not all the frames arriving at the queue enter the queue because frames are not allowed into the queue when there are already $K$ frames in the queue. Therefore, the frames are dropped with probability

$$
\begin{aligned}
P_{k} & =\rho^{k} p_{0} \\
& =\left(\frac{\lambda\left(1-p_{\text {drop }}\right)}{\mu}\right)^{K} \cdot \frac{1-\frac{\lambda\left(1-p_{\text {drop }}\right)}{\mu}}{1-\left(\frac{\lambda\left(1-p_{\text {drop }}\right)}{\mu}\right)^{K+1}}
\end{aligned}
$$

Thus, the total probability of packet loss is

$$
\begin{aligned}
P_{\text {loss }} & =P_{\text {drop }}+P_{K} \\
& =\left[1-\left(1-p_{f}\right)(1-\tau)^{n-1}\right]^{m+1}+\left(\frac{\lambda\left(1-P_{\text {drop }}\right)}{\mu}\right)^{K} \cdot \frac{1-\frac{\lambda\left(1-P_{\text {drop }}\right)}{\mu}}{1-\left(\frac{\lambda\left(1-P_{\text {drop }}\right)}{\mu}\right)^{K+1}}
\end{aligned}
$$




\subsection{Mean Delay}

The delay $D$ can be defined as the time elapsing from the instant the frame is inserted in the MAC buffer to the time in which it is successfully transmitted by receiving an acknowledgement for this frame. From this definition it is obvious that delay is associated with two factors: a medium access delay due to the number of contending stations and a queueing delay for load conditions and frame processing rates at the queue. So, the average delay is

$$
D_{\text {avg }}=D_{M A C}+D_{Q}
$$

\subsubsection{MAC Delay}

The MAC delay for a successfully transmitted packet is defined to be the time interval from the time the frame is at the head of the MAC queue ready for transmission until an acknowledgement for this packet is received. As per [16], the average MAC delay is given by

$$
E\left[D_{M A C}\right]=E[X] E[\text { slot }]
$$

Here $E[X]$ is the average number of slots spent for a successful transmission. Let STA be in the $i$ backoff stage and get the channel access with probability $c_{i}$. The average number of slots utilized by the STA in the $i$ backoff stage is $\left(C W_{i}+1\right) / 2, i \in(0, m)$ and the probability that the frame reaches the backoff stage $i$ and it is not discarded is $\frac{p_{f}^{i}-p_{f}^{m+1}}{1-p_{f}^{m+1}}, i \in(0, m)$. Hence,

$$
\begin{aligned}
E[X] & =\sum_{n=0}^{m}\left[\frac{\left(p_{f}^{i}-p_{f}^{m+1}\right)\left(\left(C W_{i}+1\right) / 2\right)}{1-p_{f}^{m+1}}\right] \\
E[s l o t] & =\left(1-p_{t r}\right) \sigma+P_{t r} P_{s}\left(1-P_{e r r}\right) T_{s}+P_{t r}\left(1-P_{s}\right) T_{c}+P_{t r} P_{s} P_{e r r} T_{e} \\
E[D] & =\sum_{n=0}^{m}\left[\frac{\left(p_{f}^{i}-p_{f}^{m+1}\right)\left(\left(C W_{i}+1\right) / 2\right)}{1-p_{f}^{m+1}}\right] \cdot\left(1-p_{t r}\right) \sigma++P_{t r} P_{s}\left(1-P_{e r r}\right) T_{s}+ \\
& +P_{t r}\left(1-P_{s}\right) T_{c}+P_{t r} P_{s} P_{e r r} T_{e}
\end{aligned}
$$




\subsubsection{Queueing Delay}

By using Little's formula [15], the expected time spent (i.e.queuing delay) in the queue can be calculated as

$$
D_{Q}=\frac{E[N]}{\lambda\left(1-P_{\text {drop }}\right)}
$$

Here $E[N]$ is the expected number of packets in the queue given by

$$
\begin{aligned}
E[N] & =\sum_{n=0}^{K} n P_{n}=\sum_{n=0}^{K} n \rho^{n} P_{0} \\
& =\rho P_{0} \frac{\mathrm{d}}{\mathrm{d} \rho}\left(\frac{1-\rho^{K+1}}{1-\rho}\right) \\
& =\rho P_{0}\left(\frac{1-\rho^{K+1}-(1-\rho)(K+1) \rho^{K}}{(1-\rho)^{2}}\right) \\
& =\left(\frac{\rho\left(1-\rho^{K+1}\right)-(1-\rho)(K+1) \rho^{K+1}}{(1-\rho)\left(1-\rho^{K+1}\right)}\right) \\
E[N] & =\left(\frac{\rho\left(1-(K+1) \rho^{K}+K \rho^{K+1}\right)}{(1-\rho)\left(1-\rho^{K+1}\right)}\right)
\end{aligned}
$$

Now using equation 28, $D_{Q}$ becomes

$$
D_{Q}=\left(\frac{\rho\left(1-(K+1) \rho^{K}+K \rho^{K+1}\right)}{(1-\rho)\left(1-\rho^{K+1}\right)}\right) \times \frac{1}{\lambda\left(1-P_{d r o p}\right)}
$$

Therefore the mean delay is given by

$$
\begin{aligned}
D & =\sum_{n=0}^{m}\left[\frac{\left(p_{f}^{i}-p_{f}^{m+1}\right)\left(\left(C W_{i}+1\right) / 2\right)}{1-p_{f}^{m+1}}\right] \cdot\left(\left(1-p_{t r}\right) \sigma+P_{t r} P_{s}\left(1-P_{e r r}\right) T_{s}+P_{t r}\left(1-P_{s}\right) T_{c}+\right. \\
& \left.+P_{t r} P_{s} P_{e r r} T_{e}\right)+\left(\frac{\rho\left(1-(K+1) \rho^{K}+K \rho^{K+1}\right)}{(1-\rho)\left(1-\rho^{K+1}\right)}\right) \times \frac{1}{\lambda\left(1-P_{\text {drop }}\right)}
\end{aligned}
$$

\section{Numerical Studies}

We study the characteristics of various IEEE 802.11n Block ACK schemes and to validate analytical model using MATLAB based numerical studies. Table 1 lists the parameters used in the numerical study. The protected Block ACK with and without reverse direction and non-protected Block ACK with and without RD are considered for comparison purposes. 
Table 1: Summary of IEEE $802.11 n$ parameters

\begin{tabular}{cccc}
\hline Payload & 1500 bytes & $\mathrm{r}$ & $2 \mathrm{Mbps}$ \\
\hline T-PHY & 192 & $\mathrm{r}^{*}$ & $1 \mathrm{Mbps}$ \\
\hline T-DATA & $192+(224+$ Payload $) / \mathrm{r}$ & Data Rate & $11 \mathrm{Mbps}$ \\
\hline T-ACK & $192+112 / \mathrm{r}^{*}$ & Block Size & 5 \\
\hline T-RTS & $192+160 / \mathrm{r}^{*}$ & Block Size RD & 3 \\
\hline T-CTS & $192+112 / \mathrm{r}^{*}$ & Fading Margin & 0.05 \\
\hline T-BAR & $192+192 / \mathrm{r}$ & Velocity & $5 \mathrm{~m} / \mathrm{s}$ \\
\hline T-BA & $192+1216 / \mathrm{r}$ & Queue Length & 50 \\
\hline
\end{tabular}

Figure 4 shows the increasing the number of STAs on channel throughput performance for a packet arrival rate of 8 pkts/sec.One can observe that in all cases throughput initially increases with the number of STAs but further increments of network size significantly reduce the throughput. This is very obvious in traditional WLANs due to collisions as the number of STAs increases in the network. Moreover, it is observed that the slope of the throughput degradation is stiffer in the case of Block ACK with RD as compared to protected Block ACK RD and in average the protection with the Block ACK mechanism provides $32.54 \%$ higher throughput. Collisions and FER rates are the dominating factors that make this difference in performance. As discussed in earlier, collisions and errors are only experienced by the initial data frames in the protected Block ACK mode. If the initial data frame is correctly transmitted then the STA will initiate the rest of the data burst where as in an unprotected scenario, (which used the Block ACK mode) there is no such protection mechanism (RTS/CTS is not considered in two way handshake scheme) resulting in a full data burst experiencing the collisions and errors (resulting in higher collision and error times). Hence, to fully utilize the RD feature of $802.11 n$, it should be integrated with the protected block ACK mechanism to achieve higher throughput. Another special observation is that, the protected block ACK scheme outperforms the RTS/CTS scheme as RTS/CTS has extra overheads.

Figure 5 shows the packet delay increases when the network size grows in 


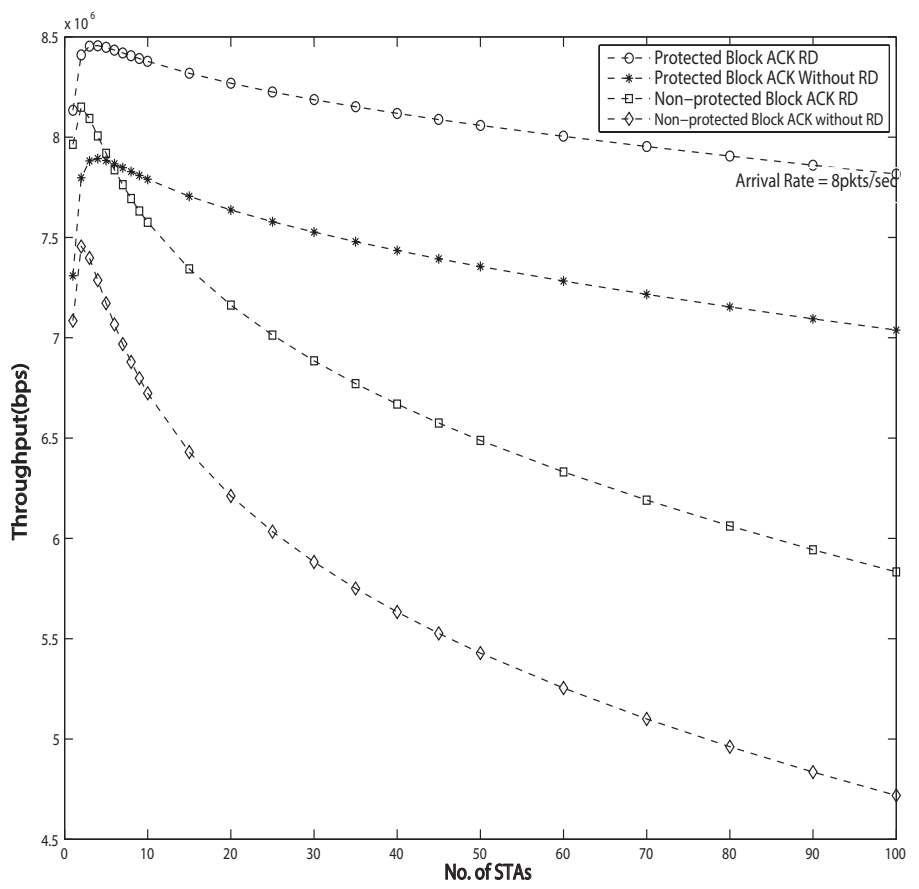

Figure 4: Channel throughput of protected Block ACK with and without RD and nonprotected block with and without RD.

all cases due to a higher number of collisions. As a large number of stations attempt to access the channel, more collisions occur, the number of retransmission increases and stations suffer longer delays. The protected Block ACK results in delays lower than that of the non-protected Block ACK scheme for both RD and without RD cases, since collisions only experienced by the first data frame in case of the protected Block ACK scheme where as the whole data burst experienced collisions in the non-protected Block ACK. However, the overhead is a little bit higher in the protected Block ACK scheme due to an additional ACK frame for the first data frame. This additional ACK frame eventually is more important to reduce the wasted time in terms of collisions or channel errors for higher data transmissions. Moreover, Fig 6 shows the variation of packet 
delay with respect to packet arrival rates. The difference of packet delay mostly remains constant between protected and non-protected Block ACK, since the packet delay contributions come from packet arrival rates and queue length as shown in Eq.29p. However packet delay is highly impacted by the number of stations given that the MAC delay's contribution become dominant.

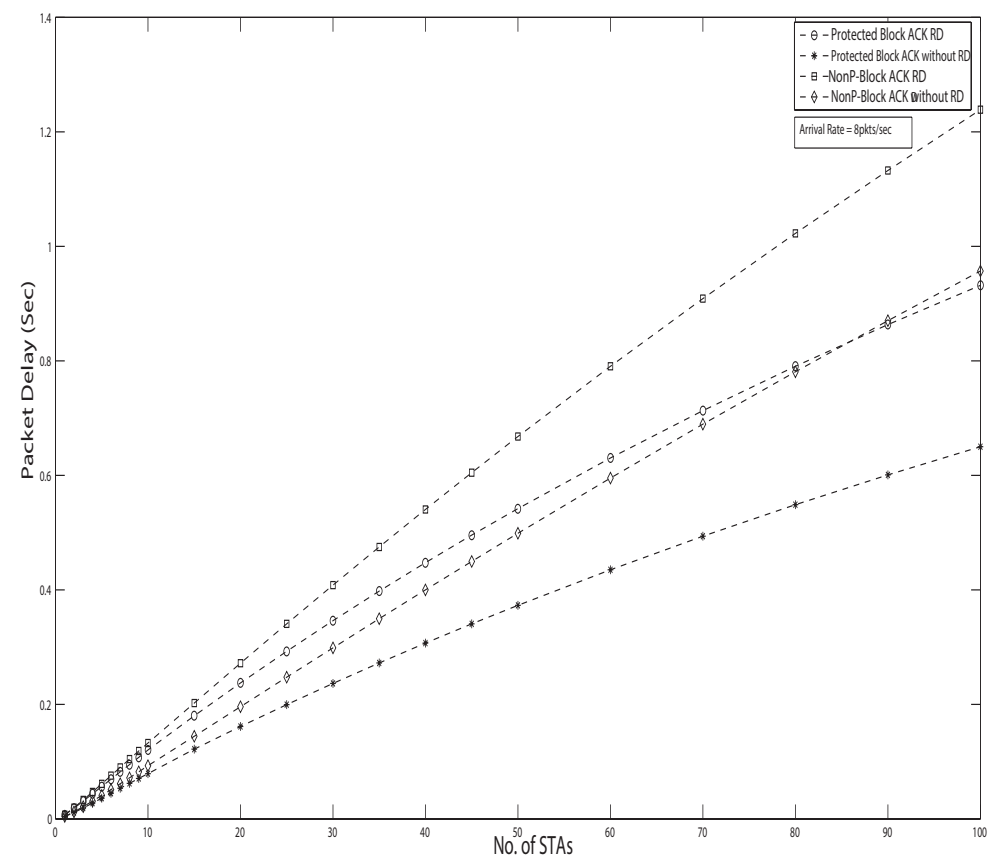

Figure 5: Packet delay versus number of stations

- The efficiency of the set of parameters used on the packet loss probability is explored in Fig 7 for protected Block ACK scheme. one can observe that shows that the choice of higher values for the contention window improve the packet loss probability by reducing the number of collisions. When $W=32, m=$ $6, m^{\prime}=5$ are used, the packet loss probability increases rapidly and is mostly exponential in nature. Now, using the same settings except a retry limit fixed to 7 , the packet drop probability decreases about $48 \%$. This is also true for 


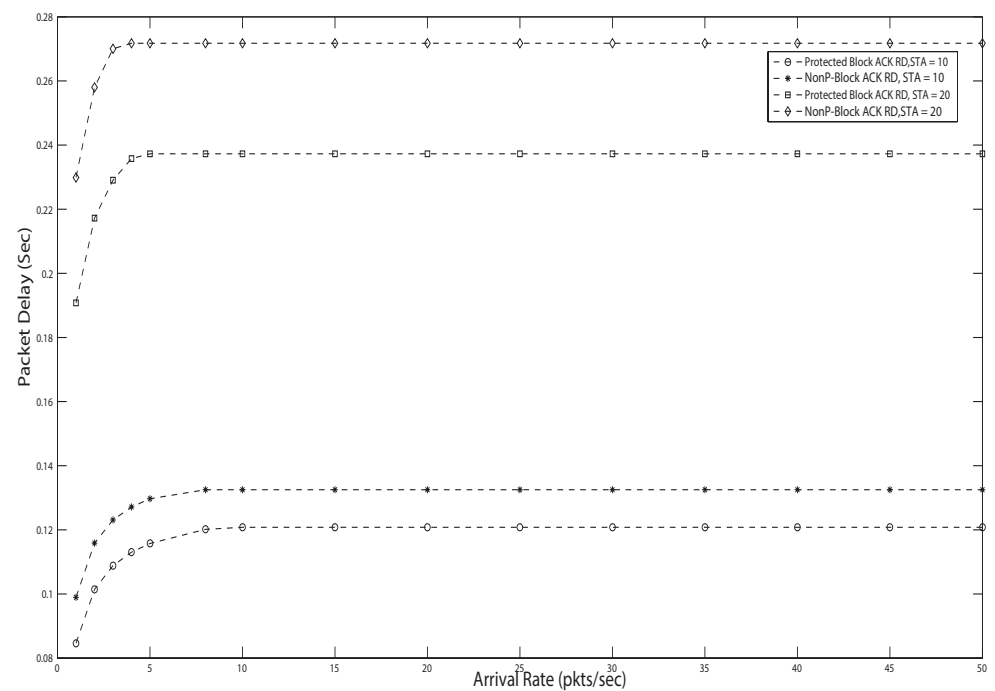

Figure 6: Packet delay versus Arrival rate.

maximum backoff stage. The packet loss probability can further be reduced by increasing contention window size from 4 to 5 . This is because the station will get more transmission opportunity according to Eq.24. However, we observe that the packet loss for the exponential backoff mechanism is smaller than the packet loss as a result of queueing mechanism (Fig 8). The probability of packet loss is almost zero for the arrival rate of $8 \mathrm{pkts} / \mathrm{sec}$ in case of the protected Block ACK with 10 stations and dramatically increases due to queue overflows. We observe similar trend for various access schemes despite of their early queue saturation.

. Figure 9 shows the probability of packet loss versus the number of stations. We observe that, the transmission probability, $\tau$ 's decreases with the number of STAs, hence increasing the probability of collisions. However, at the initial stage (for $N<5$ ) $\tau$ is increased a bit and starts decreasing. Interestingly the quantity of $\tau$ increments at the initial stage can be characterized by the packet arrival rate. It is shown that for lower packet rates (e.g. $\lambda=10 p k t s / s e c) \tau$ 


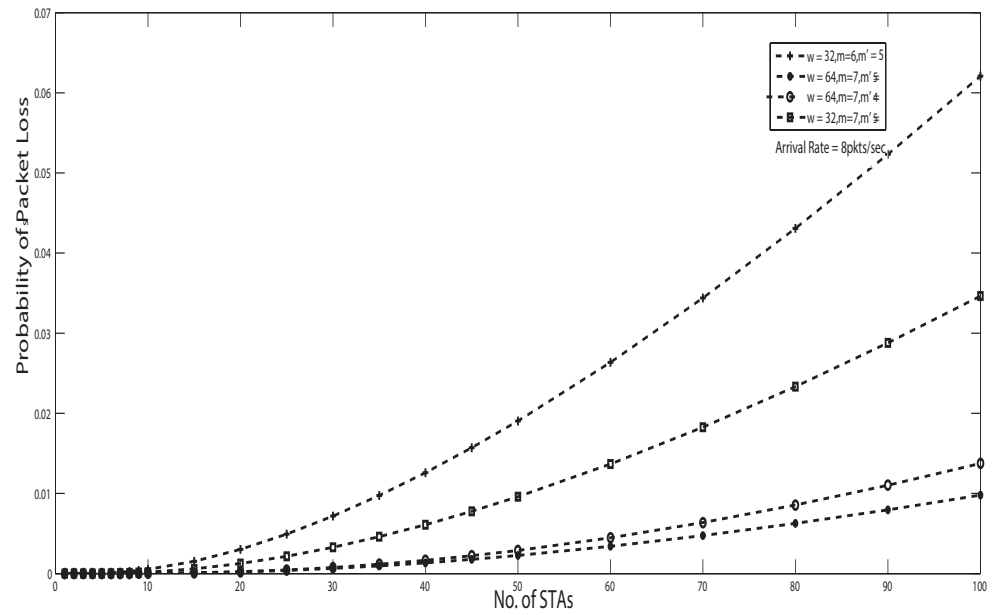

Figure 7: Probability of packet loss against the number of stations

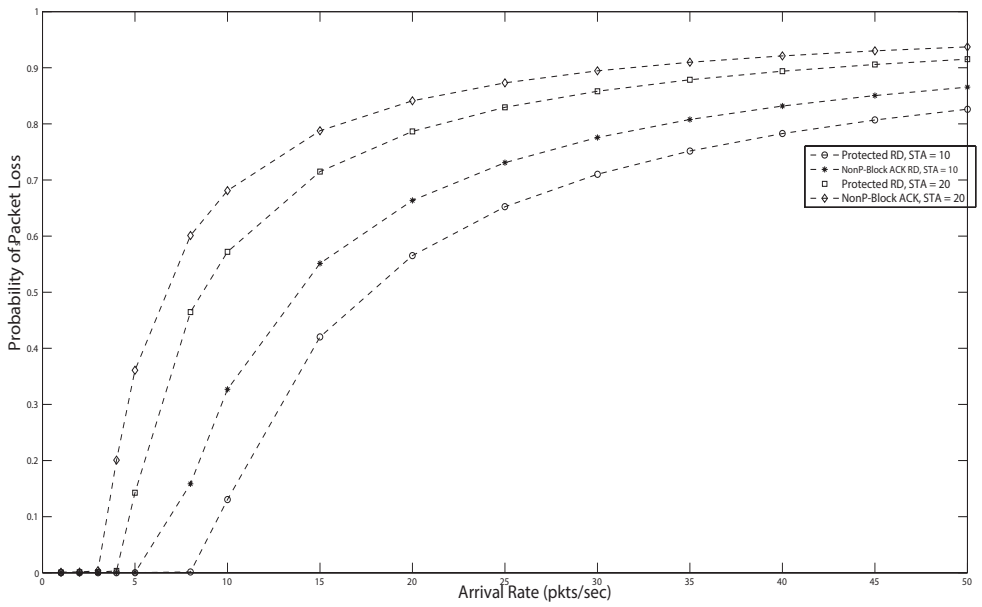

Figure 8: Probability of packet loss versus arrival rates for $N=10$ and 20 stations. Comparison of various access methods.

increases by 0.015 whereas it is only 0.005 for $\lambda=30 \mathrm{pkts} / \mathrm{sec}$.

. Figure 10 illustrates the variation of throughput with $\tau$ for both protected and non-protected Block ACK schemes. We observed that non-protected Block 


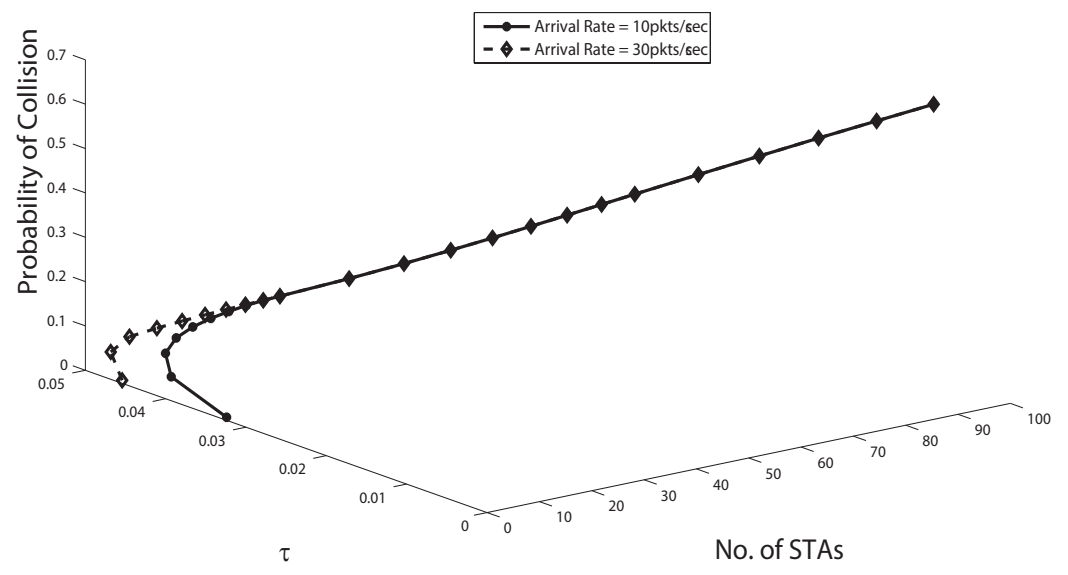

Figure 9: Relationship between probability of collision and $\tau$ for varying no. of stations.

ACK degrades performance severely when the no. of STAs is increased as a result of excessive times wasted in the channel contention. Consequently, nonprotected Block ACK scheme also suffers from higher packet delays and packet loss probability as shown in Fig. 11 and Fig. 12 , respectively.

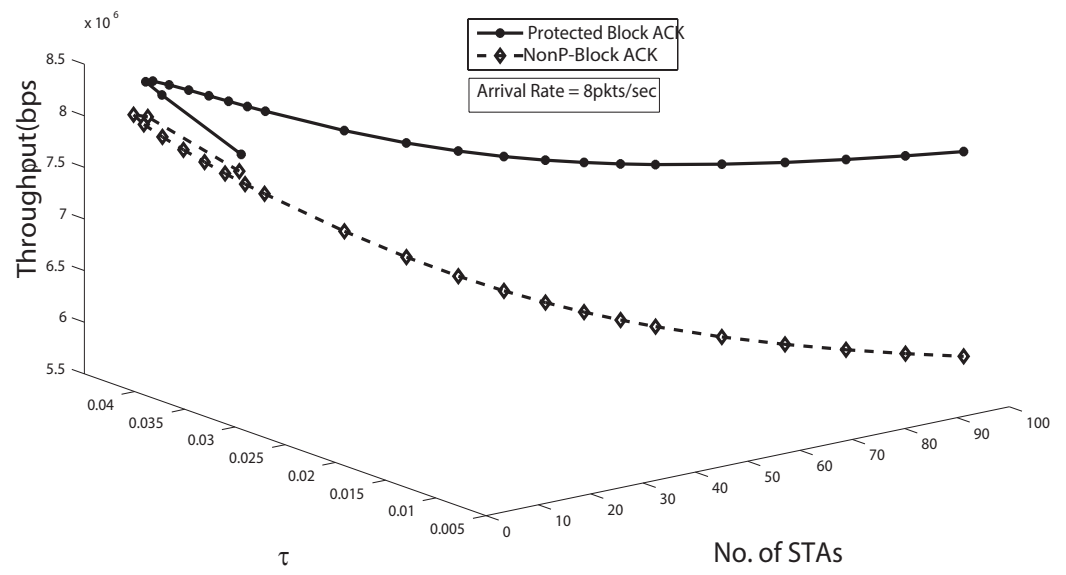

Figure 10: Relationship between Throughput and $\tau$ for varying no. of stations. 


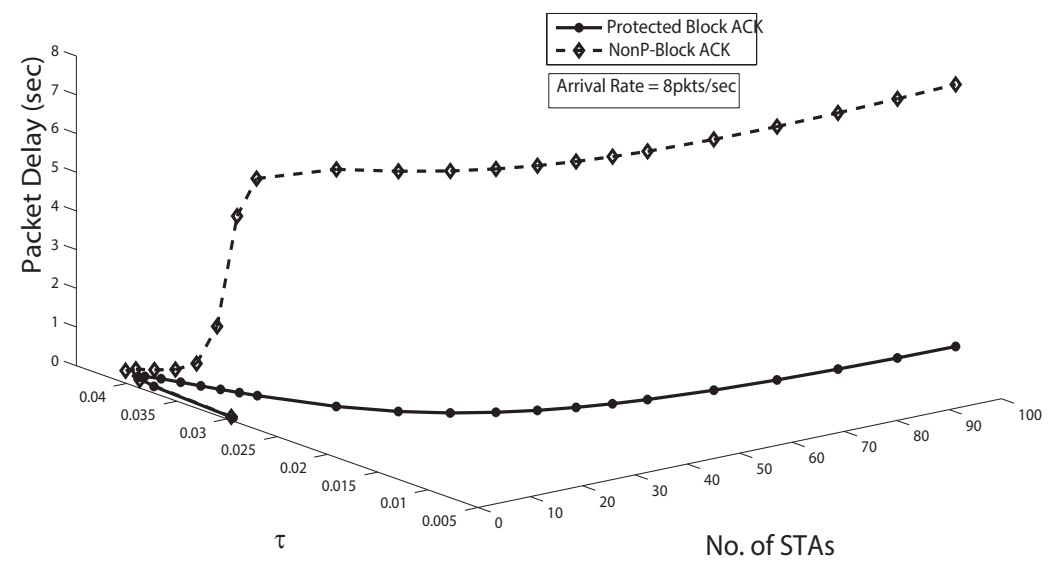

Figure 11: Relationship between packet delay and $\tau$ for varying no. of stations.

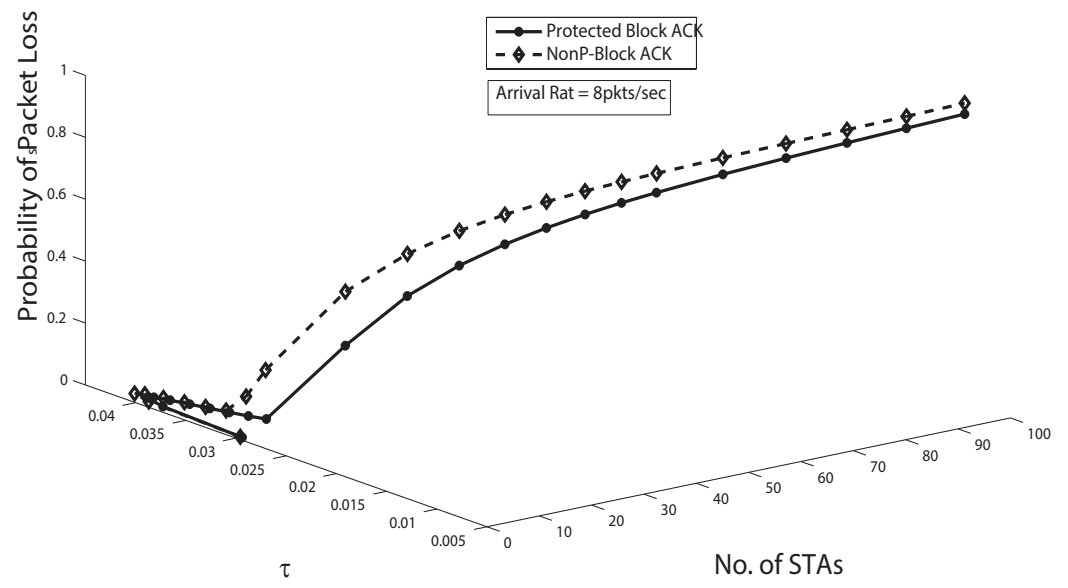

Figure 12: Relationship between probability of packet loss and $\tau$ for varying no. of stations. 


\section{Conclusion}

In this paper, we investigated the interdependencies of Block ACK and RD mechanisms for $802.11 \mathrm{n}$ using a discrete bi-directional Markov chain model under non-saturated traffic loads. We developed a mathematical model to derive system throughput, delay, and packet loss probability for both protected and unprotected Block ACK methods under various loading. The model is validated by MATLAB-based numerical studies. Results obtained have shown that the better system performance (i.e. up to $33 \%$ higher throughput and $48 \%$ less packet dropping) can be achieved using protected Block ACK in conjunction with RD data transmission. We found that 'unprotected Block ACK' wastes TXOP especially during collisions and degrades system performance significantly. To fully utilize the system performance, $802.11 n$ stations should employ protected Block ACK mechanism with RD flows. The work reported can help network planners to deploy high-speed 802.11-based networks and to contribute in the development of next generation wireless local area network 802.11ac amendment. Our future work will report on the design and performance evaluation of a crosslayer MAC protocol design supporting multimedia applications over 802.11ac. Development of an extensive simulation model to validate our numerical results presented here is also our ongoing work.

\section{References}

[1] S. Committee, "Wireless lan medium access control (mac) and physical layer (phy) specifications: Amendment 8: Medium access control (mac) quality of service enhancements," IEEE Computer Society, 2005.

[2] G. Bianchi, "Performance analysis of the ieee 802.11 distributed coordination function," IEEE Journal on Selected Areas in Communications,, vol. 18, no. 3, pp. 535-547, March 2000.

[3] F. Daneshgaran, M. Laddomada, F. Mesiti, and M. Mondin, "Unsaturated throughput analysis of ieee 802.11 in presence of non ideal transmission 
channel and capture effects," IEEE Transactions on Wireless Communications, vol. 7, no. 4, pp. 1276-1286, 2008.

[4] H. Lee, I. Tinnirello, J. Yu, and S. Choi, "Throughput and delay analysis of ieee 802.1 le block ack with channel errors," in 2nd IEEE International Conference on Communication Systems Software and Middleware. IEEE, 2007, pp. 1-7.

[5] H. Wu, Y. Peng, K. Long, S. Cheng, and J. Ma, "Performance of reliable transport protocol over ieee 802.11 wireless lan: analysis and enhancement," in proc Twenty-First Annual Joint Conference of the IEEE Computer and Communications Societies,, vol. 2. IEEE, 2002, pp. 599-607.

[6] F. Alizadeh-Shabdiz and S. Subramaniam, "A Finite Load Analytical Model for the IEEE 802.11 Distributed Coordination Function MAC," WiOpt'03: Modeling and Optimization in Mobile, Ad Hoc and Wireless Networks, p. 2 pages, Mar. 2003. [Online]. Available: https://hal.inria.fr/inria-00465993

[7] Y. S. Liaw, A. Dadej, and A. Jayasuriya, "Performance analysis of ieee 802.11 dcf under limited load," in proc. IEEE Asia-Pacific Conference on Communications. IEEE, 2005, pp. 759-763.

[8] T. Li, Q. Ni, T. Turletti, and Y. Xiao, "Performance analysis of the ieee 802.11 e block ack scheme in a noisy channel," in 2nd IEEE International Conference on Broadband Networks. IEEE, 2005, pp. 511-517.

[9] B. S. Kim, H. Hwang, and D. K. Sung, "Effect of frame aggregation on the throughput performance of ieee 802.11n," in IEEE Wireless Communications and Networking Conference, March 2008, pp. 1740-1744.

[10] V. Visoottiviseth, T. Piroonsith, and S. Siwamogsatham, "An empirical study on achievable throughputs of ieee 802.11n devices," in 7th International Symposium on Modeling and Optimization in Mobile, Ad Hoc, and Wireless Networks, 2009. WiOPT 2009., June 2009, pp. 1-6. 
[11] N. Hajlaoui, I. Jabri, and M. Ben Jemaa, "Analytical study of frame aggregation in error-prone channels," in 9th International Wireless Communications and Mobile Computing Conference, July 2013, pp. 237-242.

[12] N. Mohammad and S. Muhammad, "Modeling and analyzing mac frame aggregation techniques in $802.11 \mathrm{n}$ using bi-dimensional markovian model," in Networked Digital Technologies. Springer, 2012, pp. 408-419.

[13] D. Akhmetov, "802.11n: Performance results of reverse direction data flow," in IEEE 17th International Symposium on Personal, Indoor and Mobile Radio Communications,, Sept 2006, pp. 1-3.

[14] A. Milad, Z. Bin Muhamad Noh, A. Shibghatullah, and M. Algaet, "Design a novel reverse direction transmission using piggyback and piggyback with block ack to improving the performance of mac layer based on very high speed wireless lans," in proc. IEEE Conference on Information Communication Technologies (ICT), April 2013, pp. 263-266.

[15] L. Kleinrock, "Queueing systems, volume 1: theory," 1975.

[16] P. Chatzimisios, A. C. Boucouvalas, and V. Vitsas, "IEEE 802.11 wireless lans: performance analysis and protocol refinement," EURASIP Journal on Wireless Communications and Networking, vol. 2005, no. 1, pp. 67-78, 1900. 